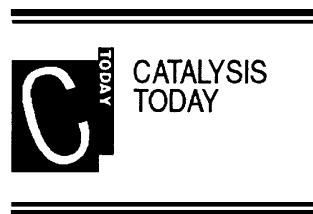

Catalysis Today 69 (2001) 365-370

www.elsevier.com/locate/cattod

\title{
Synthesis of structured filamentous zeolite materials via ZSM-5 coatings of glass fibrous supports
}

\author{
B. Louis, C. Tezel, L. Kiwi-Minsker*, A. Renken \\ Swiss Federal Institute of Technology, LGRC-EPFL, CH-1015 Lausanne, Switzerland
}

\begin{abstract}
Structured filamentous zeolitic materials were synthesized by ZSM- 5 coatings deposited on the glass fibrous supports. A homogeneous coverage of the support filaments was obtained via the synthesis of MFI crystals in fluoride-containing media at neutral $\mathrm{pH} \sim 7$. These materials combine the open macrostructure of woven filamentous fabrics with well-defined microstructure of ZSM-5 crystals. The samples were characterized by BET, XRD and SEM methods. The gas-phase partial oxidation of benzene by $\mathrm{N}_{2} \mathrm{O}$ to phenol was investigated as a function of the chemical composition of the support and the ZSM-5 synthesis procedure. The fluorinated zeolitic materials demonstrated the highest activity (above $20 \%$ of benzene conversion) with a 97\% selectivity towards phenol formation. (C) 2001 Elsevier Science B.V. All rights reserved.
\end{abstract}

Keywords: Coatings; ZSM-5; Glass fibers; Structured catalyst; Benzene hydroxylation; $\mathrm{N}_{2} \mathrm{O}$; Fluorine media

\section{Introduction}

In heterogeneous gas-phase catalytic reactions, zeolites are traditionally used in fixed-bed reactors randomly packed by powdered micro-granules or extrudated pellets. During the last decades, there has been a growing interest in catalytic reactor engineering based on structured catalytic beds [1]. Compared to traditional randomly packed fixed-bed reactors, structured catalytic beds provide improved fluid dynamics. This leads to narrower residence time distribution allowing control of consecutive reactions and resulting in increased product selectivity. Therefore, the development of novel materials suitable for a design of structured catalytic beds is warranted.

Structured zeolitic packing can be prepared via a binderless hydrothermal synthesis on metal grids [2-4]

\footnotetext{
* Corresponding author. Tel.: +41-21-693-3182;

fax: +41-21-693-3190.

E-mail address: lioubov.kiwi-minsker@epfl.ch (L. Kiwi-Minsker).
}

or ceramics [5]. In our previous publication [4], we tested the ZSM-5 coatings on metal grids for partial oxidation of benzene by $\mathrm{N}_{2} \mathrm{O}$ to phenol. Under appropriate reaction conditions at benzene/ $\mathrm{N}_{2} \mathrm{O}(\mathrm{B} / \mathrm{N})$ ratios higher than 4 , the selectivity to phenol up to $98 \%$ was achieved [4]. As metallic substrates are known to catalyze deep oxidation, the metal grid support must be completely covered by the zeolite layer, which is sometimes difficult to obtain and requires a multi-step synthesis. Even the well-covered metal supports could contribute to the deep benzene oxidation pathway: at $\mathrm{B} / \mathrm{N}<1$, selectivity towards phenol formation was observed to drop down to $75 \%$.

Another drawback of metallic supports for zeolite deposition is connected to the big difference in the dilatation coefficients of metals compared to silicates, which may cause a zeolite attrition due to its detachment under temperature changes during reactor operation. Therefore, in the present work, zeolites were deposited on amorphous silica glass supports in the form of woven fabrics. These structured supports 
combine an open macro-structure with mechanical elasticity and are not active in deep oxidation reactions [6]. Crystallization of zeolites on porous glass supports in alkaline media has already been reported [7]. The glass matrix has been partially transformed into zeolitic material and as a result the biphasic silicate material with a bimodal pore system has been obtained. The synthesis procedure described takes place in basic media at high $\mathrm{pH}$ values 11-12.5. At such $\mathrm{pH}$, the glass begins to dissolve, leading to some loss of mechanical strength of filamentous support.

Another route of the zeolite synthesis is known as "fluoride method" and uses $\mathrm{F}^{-}$instead of $\mathrm{OH}^{-}$to solubilize the reactants. The replacement of hydroxides by fluoride anions allows zeolite synthesis at low $\mathrm{pH}$ ( $\mathrm{pH}=7$ or even in slightly acidic media) [8-10].

The present work is aimed at the synthesis of filamentous structured zeolite materials via ZSM-5 crystallization on woven glass supports. Various synthetic routes in relation to the properties of the final material obtained are evaluated. The one-step benzene hydroxylation by nitrous oxide to phenol has been used for catalytic tests.

\section{Experimental}

\subsection{Support}

Two types of filamentous glass woven fabrics were used as starting material: silica glass (SGF), containing $93.5 \% \mathrm{SiO}_{2}$ and $3.5 \% \mathrm{Al}_{2} \mathrm{O}_{3}$ and E-type glass (EGF), containing 53-55\% $\mathrm{SiO}_{2}, 14-15.5 \% \mathrm{Al}_{2} \mathrm{O}_{3}$, $17.5-23 \% \mathrm{CaO}, 0.5-4.5 \% \mathrm{MgO}, 8.5 \% \mathrm{~B}_{2} \mathrm{O}_{3}$. The initial specific surface area (SSA) of the fabrics was about $1 \mathrm{~m}^{2} / \mathrm{g}$, which corresponds to the geometrical surface of the filaments. The samples of EGF were in some cases treated in aqueous solution of hydrochloric acid $(3.7 \%)$ in order to develop porosity and to increase the SSA. The acid attacks the surface of glass leaching out the non-silica phase and increasing SSA $\left(\sim 26 \mathrm{~m}^{2} / \mathrm{g}\right)$ as recently reported [6].

\subsection{Catalyst preparation}

The syntheses of structured filamentous zeolitic materials were carried out in a $250 \mathrm{ml}$ Teflon-lined stainless steel autoclave. The glass woven fabric used as a support was rolled-up and installed vertically into the autoclave, which was afterwards filled by the reaction mixture. The autoclave was put into the oven and the temperature was increased within $1 \mathrm{~h}$ up to $444 \mathrm{~K}$ and the zeolite synthesis took place during $48 \mathrm{~h}$ under autogenous pressure. In some cases, the synthesis procedure was repeated the second time with a fresh solution to enhance the weight of zeolite coating. Finally, the packing was kept in an ultrasonic bath $(45 \mathrm{kHz})$ for $20 \mathrm{~min}$ to remove loosely attached crystals and dried overnight at $400 \mathrm{~K}$.

Three synthetic routes were used for the zeolite crystallization on the filaments and are described below:

- Synthesis in alkaline media with silica source. The mixture for ZSM-5 synthesis was prepared by adding sodium aluminate $\left(52.5 \mathrm{wt} \% \mathrm{NaAlO}_{2}\right.$, Riedel-de Haën), tetrapropylammonium hydroxide (TPA-OH, 20 wt.\% in water, Fluka, Chemika) or tetrapropylammonium bromide (TPA-Br, 99\%, Merck-Suchardt) and sodium hydroxide pellets (Fluka, Chemika) in demineralized water at room temperature. Then, the tetraethyl orthosilicate (TEOS, 98\%, Merck-Suchardt) was introduced under vigorous stirring. The molar ratio was as follows: TPA-OH:Si(OEt $)_{4}: \mathrm{NaAlO}_{2}: \mathrm{Na}_{2} \mathrm{O}: \mathrm{H}_{2} \mathrm{O}=$ 8.5:100:1:28:8000. Ageing and homogenization of the mixture took place during $2-3 \mathrm{~h}$. The gel was afterwards poured in the autoclave and the procedure was performed as described above. The two-step synthesis was followed by a calcination over night at $773 \mathrm{~K}$ in air. The MFI structure was obtained in sodium form and was exchanged three times with ammonium chloride $(0.1 \mathrm{M})$ at $353 \mathrm{~K}$, then calcined at $773 \mathrm{~K}$ for $5 \mathrm{~h}$ in air resulting in H-ZSM5.

- Synthesis in alkaline media without silica source. The procedure was similar to the one described previously, but without adding a silica source in the form of $\mathrm{Si}(\mathrm{OEt})_{4}$. In this case, the silica from the support filaments was partially converted into MFI-structure. The molar ratio in the starting synthetic mixture was as follows: $\mathrm{NaAlO}_{2}: \mathrm{TPA}-\mathrm{Br}: \mathrm{Na}_{2} \mathrm{O}: \mathrm{H}_{2} \mathrm{O}=1: 56: 14: 7714$. Only one-step synthesis has been performed in this case followed by a calcination over night at $773 \mathrm{~K}$ in air. After three times ion exchange by ammonium 
chloride $(0.1 \mathrm{M})$ at $353 \mathrm{~K}$, the samples were calcined at $773 \mathrm{~K}$ for $5 \mathrm{~h}$ in air.

- Synthesis in the presence of fluoride anions (neutral media). The composition of the synthetic solution expressed in molar ratio was as follows: $\mathrm{Si}(\mathrm{OEt})_{4}: \mathrm{NaAlO}_{2}: \mathrm{TPA}-\mathrm{Br}: \mathrm{NH}_{4} \mathrm{~F}: \mathrm{H}_{2} \mathrm{O}=$ 165:1:6:183:6538. The $\mathrm{pH}$ was adjusted to 7.3 by adding a few drops of a hydrofluoric acid solution (40wt.\%). The other parameters were the same as for the synthesis in alkaline media. A cation-exchange step is not necessary in this case, since the $\mathrm{NH}_{4}$ form is directly obtained. The H-ZSM5 form was obtained simply by calcination at $773 \mathrm{~K}$ for $5 \mathrm{~h}$ in air.

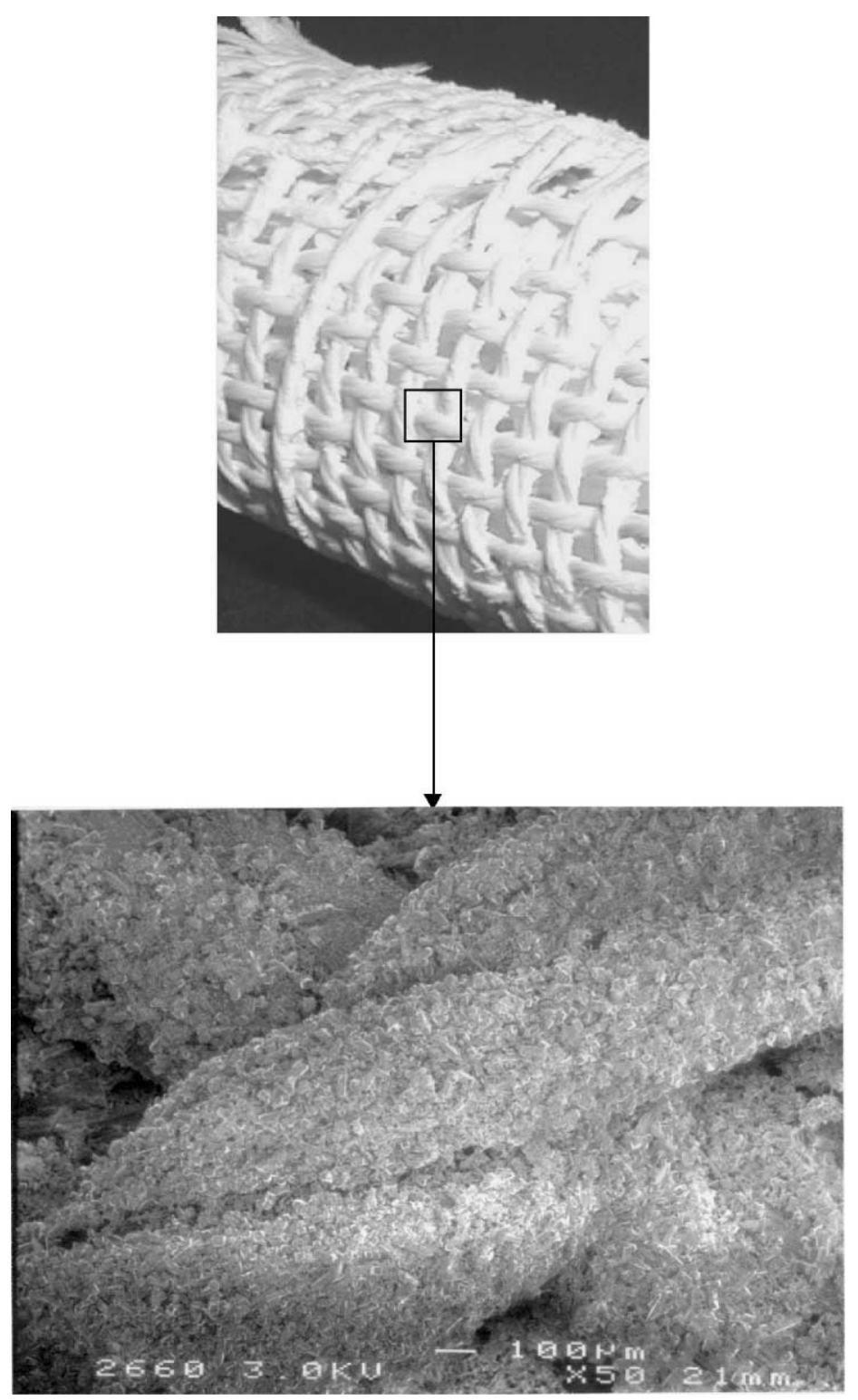

Fig. 1. Structured catalytic bed and SEM photograph of a coated thread. 


\subsection{Catalyst characterization}

SSAs of the structured catalysts synthesized were determined by nitrogen adsorption (77 K) employing BET method via Sorptomatic 1900 (Carlo-Erba). X-ray powder diffraction (XRD) patterns of catalysts were carried out on a Siemens D500 $(\theta / 2 \theta)$ diffractometer with $\mathrm{Cu} \mathrm{K} \alpha$ monochromatic radiation $(\lambda=$ $1.5406 \AA$ ). The surface morphology of the crystalline layer (zeolite coating) was studied by scanning electron microscopy on a JEOL JSM 6300F apparatus.

The catalytic activity in benzene hydroxylation by $\mathrm{N}_{2} \mathrm{O}$ was carried out at $623 \mathrm{~K}$ and atmospheric pressure in a stainless steel tubular reactor. The catalytic fabrics with ZSM-5 crystals rolled-up as shown in Fig. 1 were placed in the middle part of the reactor. Before the reaction, the catalyst was activated at $823 \mathrm{~K}$ for $1 \mathrm{~h}$ in nitrogen flow. The reaction mixture contained $3.8 \mathrm{~mol} \%$ of benzene and $16.7 \mathrm{~mol} \%$ of $\mathrm{N}_{2} \mathrm{O}$. All the gases were supplied through mass flow controllers (Bronkhorst High-Tech B.V., Ruurlo, The Netherlands). Nitrogen was used as a carrier gas and passed through a bubble column containing benzene at $20^{\circ} \mathrm{C}$ for the saturation. The total flow was set to $60 \mathrm{ml} / \mathrm{min}$ (STP). Aromatics were analyzed by GC on-line (Shimadzu GC-14A, 30 m HP-5 column, FID-detector). An IR analyzer (Siemens Ultramat-22P) monitored on-line the $\mathrm{CO}_{2}$ formation.

The activity was measured after $1 \mathrm{~h}$ on stream, since at this point, steady-state was reached. The benzene conversion was calculated from the difference in its concentration in the reactor inlet and outlet. The selectivity towards phenol was defined as the molar ratio of the phenol obtained referred to the benzene converted. The rate of benzene transformation was calculated by multiplying the difference between the benzene concentration at the reactor inlet and outlet by the total flow, referred per gram of the catalyst (support + crystals of ZSM-5 coated).

\section{Results and discussion}

\subsection{Catalyst characterization}

- Materials synthesized in alkaline media without silica source. Fig. 2 shows the SEM micrograph

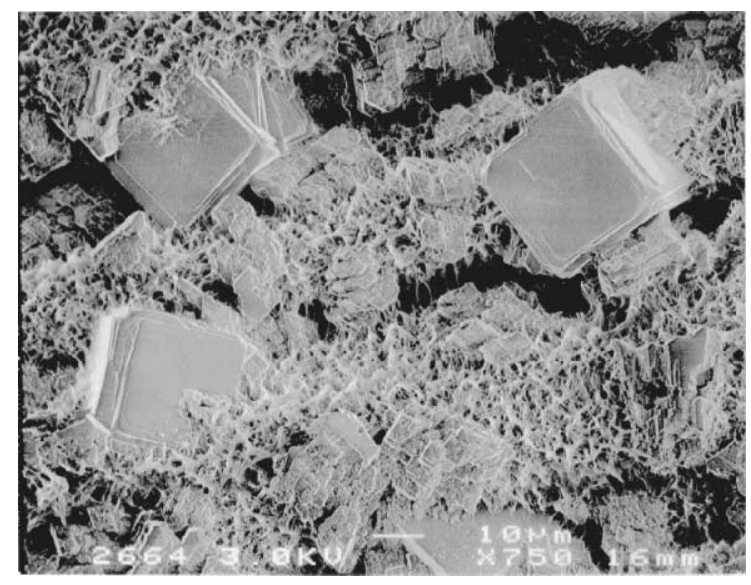

Fig. 2. SEM photograph of zeolitic coatings obtained on E-type glass fabrics in alkaline media without adding the silica source.

of the crystals grown on EGF-fiber support. The main advantage of this procedure was an absence of homogeneous zeolite crystallization in the bulk mother liquor, since no external silica source was added. Nevertheless, the cubic morphology of the crystals is clearly seen indicating the formation of a crystalline phase different from the MFI structure. The powder diffraction pattern confirms an alumina-enriched material of total composition: $\mathrm{Na}_{5.7} \mathrm{Al}_{5.7} \mathrm{Si}_{10.3} \mathrm{O}_{32} \cdot 12 \mathrm{H}_{2} \mathrm{O}$. Since the coverage remains low (Fig. 2), the material was not tested for catalytic activity.

- Synthesis in alkaline media with silica source. Both SGF and EGF filamentous supports were used for Na-ZSM- 5 crystals deposition via synthesis in alkaline media with TEOS as a silica source. The EGF support had a higher SSA of $26 \mathrm{~m}^{2} / \mathrm{g}$ compared to the SGF $\left(\sim 1 \mathrm{~m}^{2} / \mathrm{g}\right)$ and after the first synthesis step demonstrated a gain in weight of $1.8 \mathrm{~g}$, while SGF gained only $0.1 \mathrm{~g}$. The weight of the EGF catalyst (support + crystals) was again enhanced from 7.6 to $9.4 \mathrm{~g}$ after a second synthesis step.

- Fluoride method. Fig. 1 shows the abundance and the regularity of the zeolitic coverage obtained after the two-step synthesis on SGF support. The prismatic morphology of the crystals is presented in Fig. 3. The MFI structure has been confirmed by XRD. In spite of the fact that the silica source was present in the synthetic mixture, the partial 


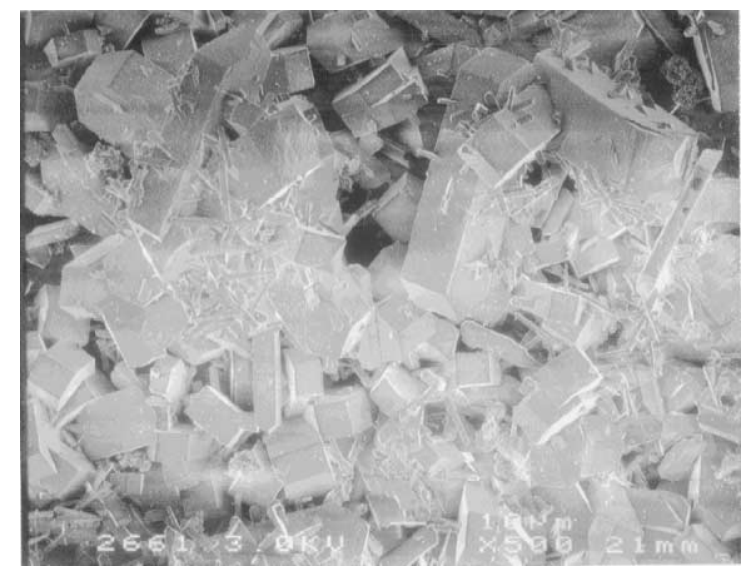

Fig. 3. Morphology of zeolite crystals obtained on E-type glass fabrics via synthesis in fluoride media.

dissolution of the support took place in the presence of fluoride anions: after the first synthetic step, a loss of weight of $6-8 \mathrm{wt} \%$ was observed. By ${ }^{19} \mathrm{~F}$ NMR it has been confirmed that silica dissolves in the fluoride-containing media producing hexafluorosilicate species [11]. It is important to note that a high coverage by zeolite was obtained on silica glass woven fabrics after two-step synthesis, while three steps are needed to reach a relatively dense coverage of metal grids [4].

\subsection{Catalytic activity}

The catalytic performance of structured ZSM-5 coatings on woven filamentous supports was tested for benzene hydroxylation. Fig. 4 shows the results obtained with different catalysts. All these materials exhibited high selectivity $(\geq 96 \%)$ towards phenol formation. The deep oxidation of aromatics was not observed over these materials even at high ratios of $\mathrm{N}_{2} \mathrm{O}$ /benzene in contrast to the metal grids used as a support.

It is clearly seen that a five times higher reaction rate was observed on the fluorine-synthesized catalysts compared to the one prepared in alkaline media. Such a big effect could not only be due to a better coverage achieved, but also due to an influence of fluorine anions present in the zeolite. In fact, Becker and

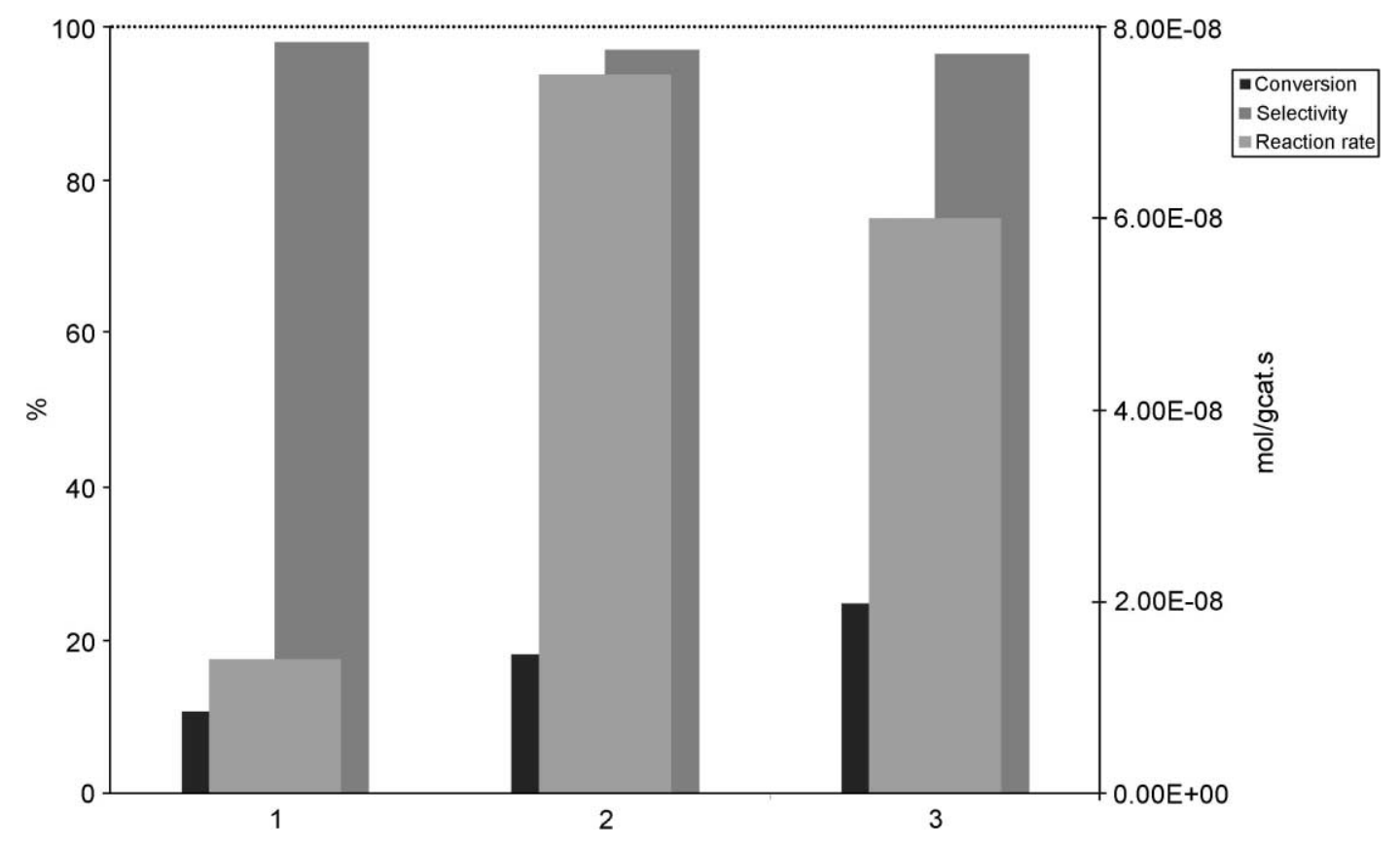

Fig. 4. Comparison of the catalytic performance in benzene partial oxidation: (1) alkaline media synthesis on EGF; (2) fluoride media synthesis on SGF; (3) fluoride media synthesis on EGF. 
Kowalak [12] observed an improved catalytic performance of F-modified zeolites. An enhanced acidity of the remaining $\mathrm{OH}$ groups by an inductive effect of neighbouring fluorine groups has been postulated, as well as the formation of new extra-framework Lewis sites (supposed to be in the form of fluoroaluminate clusters) [12]. Panov and coworkers [13,14] related the phenol formation to the high crystallinity of the MFI structure. It is known that large defect-free crystals are formed via this low $\mathrm{pH}$ procedure [9-11]. The better crystallinity of the ZSM-5 could also explain the higher activity of the catalysts 2 and 3 compared to sample 1 (see Fig. 4).

In the previous study, we reported a reaction rate of $10^{-7} \mathrm{~mol}$ of benzene converted per gram of ZSM-5 coatings on stainless steel grids [15]. It is important to note that the same order of magnitude of the reaction rate is reached (Fig. 4), but referred in this case to the total weight of the catalyst (support+ZSM-5 crystals). Furthermore, even at high nitrous oxide/benzene ratio, a 98\% selectivity towards phenol is reached on silica supports, whereas on metal grids an excess of benzene is necessary $[4,15]$.

\section{Conclusion}

- Structured filamentous zeolitic materials were synthesized by ZSM-5 crystals deposited on the glass fibrous supports via three various routes. A homogeneous coverage of the support filaments was obtained via the synthesis of MFI crystals in fluoride-containing media at neutral $\mathrm{pH} \sim 7$.

- The catalytic activity was tested in the gas-phase partial oxidation of benzene by $\mathrm{N}_{2} \mathrm{O}$ to phenol. The fluoride-containing zeolite catalysts exhibited the highest performance (benzene conversion over $20 \%$ at $98 \%$ selectivity towards phenol).

- In contrast to ZSM-5 coatings on metal grids, the selectivity is not affected at high nitrous oxide concentration at benzene $/ \mathrm{N}_{2} \mathrm{O}<1$.

\section{Acknowledgements}

Financial support from the Swiss National Foundation is gratefully acknowledged. We also thank Mr. B. Senior for technical assistance in electron microscopy measurements.

\section{References}

[1] A. Cybulski, J.A. Moulijn, Structured Catalysts and Reactors, Marcel Dekker, New York, 1998.

[2] H. van Bekkum, in: R. von Ballmoos, J.B. Higgins, M.M.J. Treacy (Eds.), Proceedings of the Ninth International Zeolite Conference, Montreal, July 5-10, Elsevier, Amsterdam, 1992, p. 247.

[3] H.P. Calis, A.W. Gerritsen, C.W. van den Bleek, C.H. Legein, J.C. Jansen, H. van Bekkum, Can. J. Chem. Eng. 73 (1995) 120.

[4] B. Louis, P. Reuse, L. Kiwi-Minsker, A. Renken, Appl. Catal. A 210 (2001) 103.

[5] R. Lai, Y. Yan, G.R. Gavalas, Micropor. Mater. 37 (2000) 9.

[6] L. Kiwi-Minsker, I. Yuranov, B. Siebenhaar, A. Renken, Catal. Today 54 (1999) 39.

[7] W. Schwieger, M. Rauscher, R. Mönnig, F. Scheffler, D. Freude, Stud. Surf. Sci. Catal. 129 (2000) 121.

[8] J.L. Guth, H. Kessler, M. Bourgogne, French Patent No. 2564451 (1984).

[9] H. Kessler, J. Patarin, C. Schott-Darie, Stud. Surf. Sci. Catal. 85 (1994) 75.

[10] J.L. Guth, H. Kessler, J.M. Higel, J.M. Lamblin, J. Patarin, A. Seive, J.M. Chezeau, R. Wey, Zeolite synthesis in the presence of fluoride ions, American Chemical Society Symposium, 1989, p. 176.

[11] S.A. Axon, J. Klinowski, Appl. Catal. A 111 (1994) 29.

[12] K.A. Becker, S. Kowalak, J. Chem. Soc., Faraday Trans. I 83 (1987) 535.

[13] L.V. Piryutko, O.O. Parenago, B.V. Lunina, A.S. Kharitonov, L.G. Okkel, G.I. Panov, React. Kinet. Catal. Lett. 52 (1994) 275.

[14] A.S. Kharitonov, V.B. Fenelonov, T.P. Voskresenskaya, N.A. Rudina, V.V. Molchanov, L.M. Plyasova, G.I. Panov, Zeolites 15 (1995) 253.

[15] B. Louis, L. Kiwi-Minsker, P. Reuse, A. Renken, Ind. Eng. Chem. Res. 40 (2001) 1454. 